\title{
Robust watermarking scheme based LWT and SVD using artificial bee colony optimization
}

\author{
Adnan Mohsin Abdulazeez ${ }^{1}$, Dilan M. Hajy ${ }^{2}$, Diyar Qader Zeebaree ${ }^{3}$, Dilovan Asaad Zebari ${ }^{4}$ \\ ${ }^{1}$ Presidency of Duhok Polytechnic University, Duhok, Kurdistan Region, Iraq \\ 2,3,4 Research Center of Duhok Polytechnic University, Duhok, Kurdistan Region, Iraq
}

\begin{abstract}
Article Info
Article history:

Received Apr 29, 2020

Revised Jun 15, 2020

Accepted Jun 27, 2020

\section{Keywords:}

Encryption

Image watermarking

Lifting wavelet transform

Multi-objective artificial bee

colony optimization

Multiple scaling factor

SVD

ABSTRACT

This paper proposes a watermarking method for grayscale images, in which lifting wavelet transform and singular value decomposition are exploited based on multi-objective artificial bee colony optimization to produce a robust watermarking method. Furthermore, for increasing security encryption of the watermark is done prior to the embedding operation. In the proposed scheme, the actual image is altered to four sub-band over three levels of lifting wavelet transform then the singular value of the watermark image is embedded to the singular value of LH sub-band of the transformed original image. In the embedding operation, multiple scaling factors are utilized on behalf of the single scaling element to get the maximum probable robustness without changing watermark lucidity. Multi-objective artificial bee colony optimization is utilized for the determination of the optimal values for multiple scaling components, which are examined against various types of attacks. For making the proposed scheme more secure, the watermark is encrypted chaotically by logistic chaotic encryption before embedding it to the host (original) image. The experimental results show excellent imperceptibility and good resiliency against a wide range of image processing attacks.
\end{abstract}

This is an open access article under the CC BY-SA license.

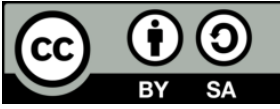

\section{Corresponding Author:}

Diyar Qader Zeebaree

Research Center

Duhok Polytechnic University

Email: Dqszeebaree@dpu.edu.krd

\section{INTRODUCTION}

The organization of determining copyright ownership and proving the novelty of digital content are widely required for the audio, video, and multimedia creations as they were swiftly disseminated over the rapid dissemination system such as the internet and satellite. Because they can be so easily modified, copied and illegally attacked by anyone, new technology is needed to secure them. Digital watermarking is one of the most common and efficient approaches that is considered as an instrument for providing the copyright defense of digital content and has been proposed as a valid solution for this problem [1-4]. The watermarking is based on embedding a signature or more information into the host signal for identifying the copyright ownership [5-8]. Digital watermarking conceals the copyright data into the digital data via an algorithm. The hidden particulars to be embedded can be some words, writer 's serial digits, organization logo, photos containing exceptional influence. These secret details are embedded in digital information (images, audio, and video) to uphold the security, data authentication, identification of possessor and copyright defense [913]. The image that is required to be embedded is called a watermark image and the image which carries the watermark is called host or original image. Watermarked image is a combination of original and watermark image which is obtained from watermarking process [14] as displayed in Figure 1. 


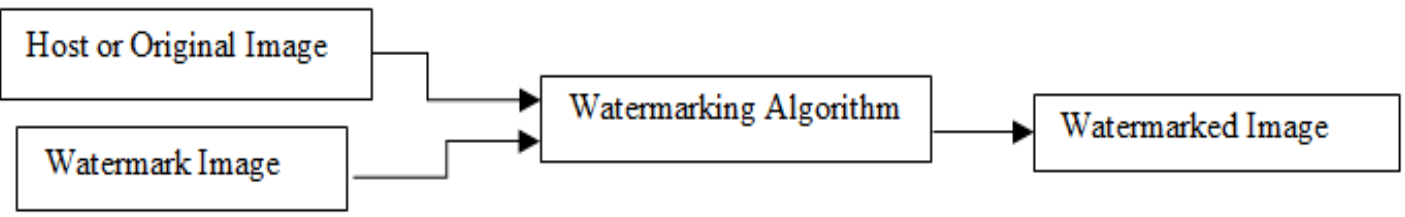

Figure 1. Watermarking process

The watermarking process have some requirements, the more important are: robustness, imperceptibility, and transparency [1]. Robustness is the strength of the watermarking system against hacker's attacks or usual image processing activities like cropping, noise, rotation, and compression. One of the most significant determinants for assessing the operation of the watermarking algorithm is the imperceptibility of watermark that is established by the Peak Signal to Noise Ratio (PSNR). It means the perceptual difference linking the watermarked and the actual records should be insignificant to the human eye [9]. Furthermore, to achieve transparency, the quality of the watermark and actual reflection should not be affected by the watermarking process [15]. Digital watermarking is applicable in the spatial and transform spheres for the achievement of robustness and imperceptibility. In spatial domain procedures, the watermark is embedded straight to the pixel area; making implementation easier but lacks robustness. While in transform domain procedures to coefficients then fix watermark into it. Then, to obtain embedded image inverse transform is applied. It possesses further robustness, fewer command of endless quality and mostly is acceptable for copyright application. The most regularly utilized methods are Discrete Cosine Transform (DCT) domain [16], Discrete Wavelet Transform (DWT) [17], Singular Value Decomposition (SVD) [18] and Lifting Wavelet Transform (LWT) [19, 20]. Transforming domain techniques are currently more extensive due to now come into more widely used as they always have good robustness to common image processing. While these procedures entail embedding the watermark in the host's transform domain, in comparison to spatial, their sophistication, robustness, and popularity are high [21]. The lifting method is a procedure for establishing both the wavelets and performing the DWT. There is value in merging the procedure and designing of the wavelet filters while executing the wavelet transform, hereafter referred to as the second generation wavelet transform. The procedure was instituted by Wim Sweldens [22].

The LWT has special advantages over the traditional first-generation wavelets [22]. Furthermore, the SVD is suitable for watermarking since slight change in singular value doesn't influence the quality of the image [18]. Using LWT combined with SVD leads to simplification of watermark recovery, enhances the robustness of the watermark and spreads the watermark throughout the spectrum [19]. Generally, the watermark can be balanced by a Scaling Factor (SF) utilized to manage the sturdiness of the watermark. For some SVD-based researches, the scaling factor is constant. Nevertheless, arguments imply that the consideration of a sole and stable scaling factor is inapplicable [23]. The execution of the watermarking activity is highly dependent on adopting a proper SF. A higher SF implies high-quality distortion of the host image (transparency) and the powerful the robustness. Even so, a lower SF translates to enhanced image quality and the weaker the robustness [24]. The incorporation of Multiple Scaling Factors (MSF) rather than a Single Scaling Factor (SSF) has higher applicability for modifying all the pixel estimates of the actual picture [25]. For watermarking, the determination of MSFs estimates is a difficult problem which is optimization problem. Multi-Objective Artificial Bee Colony (MOABC) algorithm can be used to provide a solution to such a problem. The algorithm replicates the intelligent foraging character of honeybee swarms. It is a very straightforward, robust and population-based stochastic optimization algorithm [26].

\section{LITERATURE REVIEW}

In 2009 Khaled Loukhaoukha and Jean-Yves Chouinard [27], suggested hybrid robust digital watermarking technique depending on SVD and LWT. In this algorithm the cover picture is transformed using 2-level LWT, a sub-band is selected, and inverse lifting is performed to that subband. Then, the singular estimate of watermark image is embedded to singular estimate of that sub-band, at the end the image is reconstructed. The proposed method improves the operation of the watermarking algorithm established on the joint LWT and SVD and shows that the imperceptibility is provided as well as greater robustness against common signal processing. In 2011 Khaled Loukhaoukha et. al. [25], presented a robust watermarking algorithm established on LWT and SVD using MSFs optimized by multi-objective ant colony optimization (MOACO). The singular estimates of the binary watermark are embedded in a detail sub-band of host picture. For gaining the top probable robustness minus dropping watermark transparency, MSF is utilized as an alternative for SSF. Establishing the optimal estimates of the MSF is done by using MOACO. This

Robust watermarking scheme based LWT and SVD using artificial bee colony... (Adnan Mohsin Abdulazeez) 
watermarking scheme surpasses SSF watermarking procedures in relation to imperceptibility and robustness. Also, the problem of incorrect certain detections which influences most SVD-watermarking algorithms is settled by one-way hash functions and watermark encryption. In 2012 Sushma G. Kejgir and Manesh Kokare [9], proposed a watermarking procedure based on LWT and SVD. In this method, the original image is transformed using LWT into sub-bands. Intensities of sub-bands are compared to the computed "Q-value", the sub-band possessing energy higher in comparison to the calculated "Q-value" is chosen for watermark embedding. SVD matrix is obtained for this sub-band and utilized to embed the gray level digital signature as a watermark. The results of this technique show advantages over techniques that use DWT instead of LWT. In 2012 Yongchang Chen and et. al.[21], proposed a watermarking scheme established on SVD and DWT with an artificial bee colony algorithm (ABC). In this scheme the similarity measure of U-matrix for ownership is checked to solve the problem of false position detection. To obtain the greatest feasible robustness without dropping the transparency, an adaptive scaling factor is obtained by the ABC algorithm.

\subsection{Wavelets and lifting scheme}

Wavelet transform (WT) is one of the most popular frequency domain examples [22,23]. Wavelet transfonn has been widely studied in many aspects of image processing [24]. Wavelets are a flexible instrument for portraying common functions or data sets. They can be thought of as data establishing blocks. They have an essential trait of enabling effective representations that can be calculated with speed. That means they have the capability to quickly seizure the data set extracts using minimal coefficients. This is as a result of the correlation available in many data sets both in time and frequency [25]. The lifting method is a popular procedure used in biorthogonal wavelets. It is a simple but quite powerful tool for the construction of second-generation and allows for the efficient implementation of integer wavelet transforms [25]. It is not only restricted to one-dimensional signals; it can also be used for two-dimensional signals [28]. The lifting procedure is a method for not only scheming wavelets but also doing wavelet transform. The forward lifting technique splits the provided data set being handled into even half and odd half [9]. It is important to link the phases and device the wavelet filters during wavelet modification, referred to as "second-generation wavelet transform".

The discrete wavelet transform uses many filters distinctly to the same signal. On the contrary, the signal is split like a zipper and further several convolution-accumulate processes are utilized for the lifting scheme [29]. The lifting scheme is an efficient implementation of a wavelet transform algorithm. The initial development of this technique was for enhancement of the wavelet transform but later expansion was made to a generic technique to establish the so-called second-generation wavelets (that are wavelets that do not automatically utilize similar function prototype at various levels). There are greater flexibility and much power to the second-generation wavelets in comparison to the first-generation wavelets. The lifting scheme is an application of the filtering procedures at every phase [30]. The most noticeable advantages of lifting schemes are simple structure, reduction of distortion and aliasing effects, fast and in-place computation of wavelet transform, that is there is no need for additional auxiliary memory [25]; Therefore, it is widely utilized in signal processing. A classic lifting phase entails three procedures, namely a split, a predict, and an update operation. Forward lifting and inverse lifting stages are exhibited in Figure 1 and Figure 2, separately.

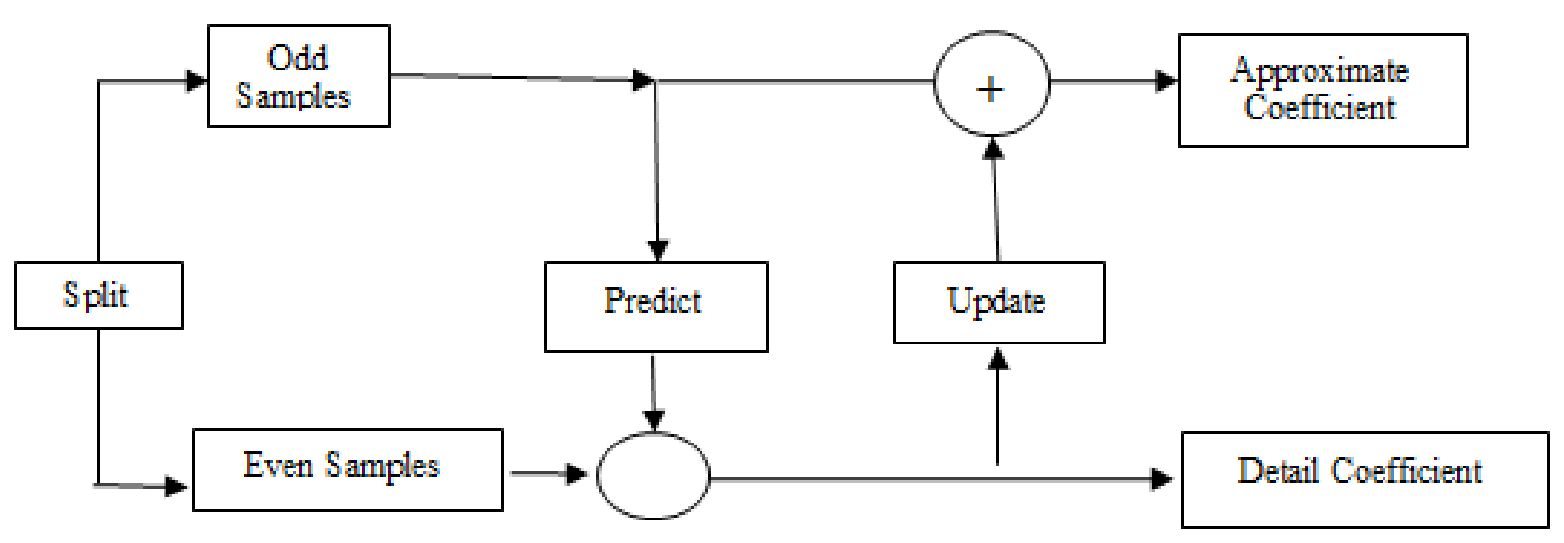

Figure 2. Lifting scheme forward wavelet transform 
In lifting wavelet transform shown in Figure 3, the signal or image is split into two separate sets the first is called even samples and the second is called odd sample establishing two vertical halves (L-band Hband separately) [29]. Then the predicate step is applied to get detail coefficients and these coefficients are used in the update step to get approximate coefficients. The process of decomposition into four bands of data, which are approximation coefficients matrix (LL) and detail coefficients matrices HL, LH, HH Figure 4(a). The decompositions are replicated on the estimation coefficients up to a level Figures 4(b) and 4(c). Suitability of the wavelet transform is not possible for pictures containing vast differences because of lack of detail decomposition the high levels with further description by the small-scale wavelet coefficients.

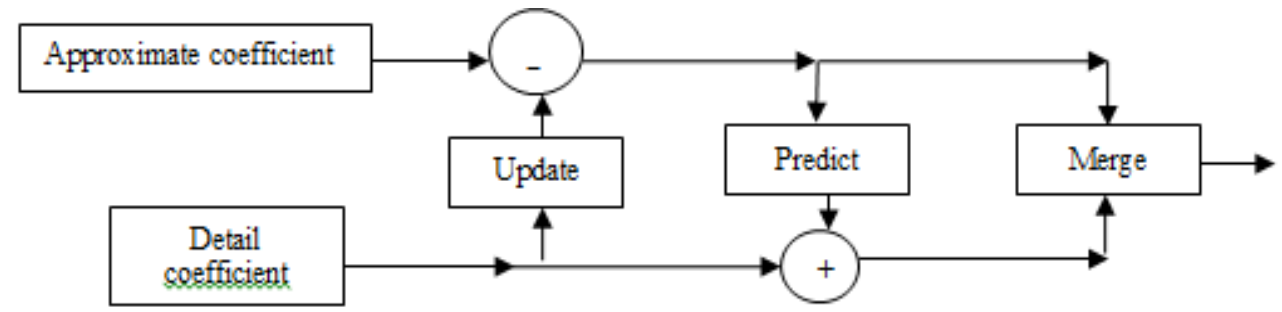

Figure 3. Inverse lifting scheme wavelet transform

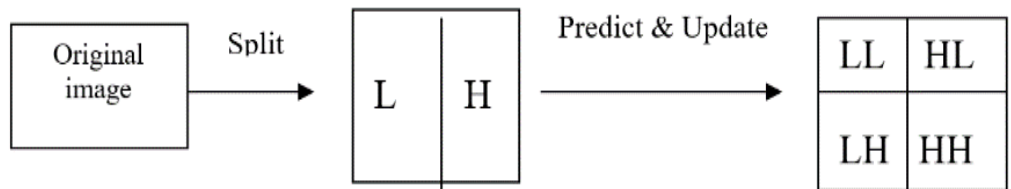

(a) First Level of Decomposition

\begin{tabular}{|l|l|l|}
\hline $\mathrm{LL} 2$ & $\mathrm{HL} 2$ & \\
\cline { 1 - 1 } $\mathrm{LH} 2$ & $\mathrm{HH} 2$ & \\
\cline { 1 - 1 } $\mathrm{LL} 1$ & \\
$\mathrm{LH} 1$ & $\mathrm{HH} 1$ \\
\hline
\end{tabular}

(b) Second Level of Decomposition

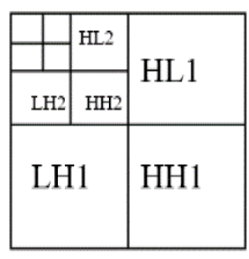

(c)Third Level of Decomposition

Figure 4. An image decomposition over three level

Generally, the concentration of much of the picture energy is at the low-frequency sub-bands LL degrading the picture remarkably. Enhancement of robustness is possible via hiding in the low-frequency sub-bands [15]. Several wavelet-domain watermarking procedures entail embedding of the watermark into the middle-frequency sub-bands coefficients for two rationalities: first, low-frequency components possess greater effects on the image quality in comparison to the middle and high-frequency elements; two, highfrequency elements are easily eliminated after low pass filtering [31].

\section{SINGULAR VALUE DECOMPOSITION}

A single value of image decomposition is considered as a general linear algebra, where a given matrix means the image, in this case, is diagonalized such that its single energy is localized in a few of single values mostly. Being among the most powerful analysis techniques of linear algebra, SVD contains stability and effectiveness for splitting the structure into a collection of linearly independent constituents, every one of them containing individual energy presentation [32], it packs maximum signal energy into, as possible, minimal coefficients. SVD has been substantiated to be an efficient instrument for signal processing techniques like image coding, signal enhancement, and image filtering. In watermarking, SVD is widely used because of the advantage it states to hide the watermark effectively when changes occur in large singular values [33]. Every actual matrix M can be decomposed by SVD into a product of 3 matrices [21] as shown in Figure 5. 


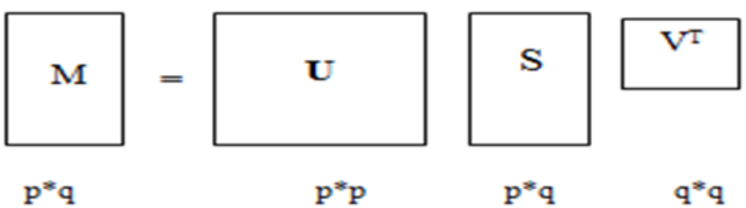

Figure 5. Illustration of Decomposing M to USVT

Where Matrix $U$ is an $p \times p$ orthogonal matrix,

$\mathrm{U}=[\mathrm{u} 1, \mathrm{u} 2, \ldots \mathrm{u} \mathrm{r}, \mathrm{ur}+1, \ldots, \mathrm{up}]$

Column vectors $u_{i}$, for $\mathrm{i}=1,2, \ldots, \mathrm{p}$, form an orthonormal set:

$u_{i}^{t}=\left\{\begin{array}{l}1 \ldots \ldots \ldots i=j \\ 0 \ldots \ldots i \neq j\end{array}\right.$

Where Matrix V is an $q \times q$ orthogonal matrix,

$\mathrm{V}=[\mathrm{v} 1, \mathrm{v} 2, \ldots \mathrm{vr}, \mathrm{vr}+1, \ldots, \mathrm{vq}]$

Column vectors $\mathrm{v}_{\mathrm{i}}$, for $\mathrm{i}=1,2, \ldots, \mathrm{q}$, form an orthonormal set:

$v_{i}^{T} v=\left\{\begin{array}{l}1 \ldots \ldots i=j \\ 0 \ldots \ldots i \neq j\end{array}\right.$

$\mathrm{S}$ is an $\mathrm{p} \times \mathrm{q}$ diagonal matrix with singular values $(\mathrm{SV})$ on the diagonal. The matrix $\mathrm{S}$ can be shown as in follow:

$\mathrm{S}=\left[\begin{array}{ccccccc}\sigma_{1} & 0 & \ldots & 0 & 0 & \ldots & 0 \\ 0 & \sigma_{2} & \ldots & 0 & 0 & \ldots & 0 \\ \vdots & \vdots & \ldots & \vdots & \vdots & \ldots & \vdots \\ 0 & 0 & \ldots & \sigma_{\mathrm{r}} & 0 & \ldots & 0 \\ 0 & 0 & \ldots & 0 & \sigma_{\mathrm{r}+1} & \ldots & 0 \\ \vdots & \vdots & \ldots & \vdots & \vdots & \ldots & \vdots \\ 0 & 0 & \ldots & 0 & 0 & \ldots & 0 \\ 0 & 0 & \ldots & 0 & 0 & \ldots & \sigma_{\mathrm{q}}\end{array}\right]$

$\mathrm{S}=\operatorname{diag}\left(\sigma_{1}, \sigma_{2}, \ldots, \sigma_{\mathrm{q}}\right)$ satisfies $\sigma_{1} \geq \sigma_{1} \geq \cdots \sigma_{1} \geq \sigma_{\mathrm{r}+1} \ldots \geq \sigma_{\mathrm{q}} \geq 0$

The diagonal items of $\mathrm{S}$ are termed as the singular values of $\mathrm{M}$, the column vectors of $\mathrm{U}$ are termed as the left singular vectors of $\mathrm{M}$, and $\mathrm{V}$ is referred to as the right singular vectors of $\mathrm{M}$. Every singular value details the luminance (brightness) of a picture layer with the equivalent set of singular vectors indicating the geometry of the picture layer [21].

During image transformation using SVD, compression does not take place, rather the details occupy a form where the initial singular value has a higher quantity of the image details. This entails utilization of minimal singular values to show the image with less contrast in comparison to the actual [24]. Many SVD characteristics are very significant for pictures like; its greatest energy packing, resolving of the least-squares issue, calculating pseudo- inverse of a matrix and multivariate analysis [32].

Some attracted attention properties that influence watermarking are mentioned below:

a) Few singular values (SVs) can constitute a large section of signal energy [23].

b) The SV's of an image possesses great stability, that is, with little addition of perturbation to a picture, there is no significant alteration to its Singular values [34]. In other words, little variations of singular values have no notable influence on the quality of the cover picture.

c) The largest of the modified SV's against signal processing attacks change very little, because of perfect noise immunity of the SV's [35]. 


\subsection{Artificial bee colony (ABC)}

By Dervis Karaboga in 2005 the $\mathrm{ABC}$ algorithm is among the many current explained, prompted by the clever character of honey bees [36]. It is easy like both algorithms Particle Swarm Optimization (PSO) and Differential Evolution (DE) and utilizes usual control variables like the size of colony and maximum number of cycles. $\mathrm{ABC}$ as an optimization instrument supplies a population-based search technique where individuals termed as locations are altered by the artificial bees with time and the bee's project in discovering the food source areas with more nectar amount and lastly the one with the greatest nectar [37-45] Karaboga [36] suggested the $\mathrm{ABC}$ algorithm prompted by this foraging character of honeybees. This algorithm considers an area that provides food considered as a candidate outcome for the problem of optimization and the outcome of the fitness is constituted by the quantity of nectar in the food origin. Comparable to the real bee colony is the artificial bees comprising of employed bees, Onlookers and scouts. In ABC, employed bees cover half of the population while the other half are onlookers. The assumption is that the quantity of food origin is equal to the number of employed bees. After abandoning a food origin, the employed bee of that food origin becomes a scout and performs a random search.

As it's described in [45], The algorithm generally outperformed other techniques (SIMPSA, NE SIMPSA, GA, ANTS) that were compared with it in relation to how fast optimization and accuracy the results can be established. The $\mathrm{ABC}$ algorithm is so easy in comparison with other prevailing swarm-based algorithms. Hence, W.Zou and et. al. [26] developed ABC to handle multi-objective optimization issues. In this algorithm, all solutions are food source areas like all bees are considered as onlooker bees, with no employed bees and scouts.

In MOABC an external archive is utilized to store past vectors found along the search procedure. In every generation, each observer randomly selects a food origin from an outer log, proceeds to the food origin place, and selects a current food origin. In the initialization phase after producing food source positions randomly, the fitness of these positions is evaluated and are stored in external archive EA. In the onlooker bees' phase, a detailed learning procedure is utilized for the production of new solution vi. Every bee xi haphazardly selects $\mathrm{m}$ dimensions and discovers from an infusion that is haphazardly chosen from EA. The production of the current infusion is by utilizing the succeeding expression:

$$
\mathrm{v}_{\mathrm{ij}}=\mathrm{x}_{\mathrm{if}_{\mathrm{m}}}+\Phi(\mathrm{m})\left(\mathrm{EA}_{\mathrm{kf}}-\mathrm{x}_{\mathrm{if}}\right)
$$

where $\mathrm{k} \in(1,2, \ldots \mathrm{p})$ is index selection randomly, $\mathrm{p}$ represents the solutions number in the EA. $\mathrm{f}_{\mathrm{m}}$ indicates random permutation of first integer numbers $1: n$, and $f(m)$ represents which dimension of $x_{i}$ 's should learn from $\mathrm{EA}_{\mathrm{k}}$. As dissent to $\Phi \mathrm{ij}$ in original $\mathrm{ABC}$ technique, $\Phi_{\mathrm{ij}}$ generate random numbers where all numbers are between $[0,2]$. This modification makes the potential search space around EAk.

After generating a new solution, the fitness is calculated then the mechanism of greedy chosen is applied to determine which solution should enter EA. After each generation, where the allocated size of EA has been exceeded by the number of solutions, congesting distance is utilized to omit the crowded members. Sorting population in the external archive from the function value of each objective in ascending order is needed to compute the crowding distance. After that, the function of each objective, solutions of the boundary with function values (smallest and largest) are employed as infinite values of distance. However, all other solutions (intermediate) are employed as a value of distance equal to the absolute normalized various in the function values of two neighboring solutions. The process of this computation is persistent with functions from other objectives. Overall, the value of crowding distance is computed as a summation of individual distance values corresponding to the values of each objective. The function of each objective is normalized before computing the crowding distance $[31,46]$.

\section{PROPOSED SCHEME}

In general, reconstruction of images by lifting wavelet transform is good compared with other general wavelet transforms, because it confirms smoothness ad reduce aliasing effects. Using LWT increases the robustness of embedded watermark in cover image, reduces loss of information and helps to recover watermark. About SVD, it's stability and effectiveness enable splitting the system into a pair of linearly independent elements. So, watermarking scheme based on SVD is advantageous since slight modifications in the singular values lacks significant effect on the quality of image. Therefore, the proposed scheme combines the properties of both LWT and SVD.

This scheme uses LWT to decompose the object image into four sub-bands (LL, LH, HL, HH). Generally, most of the energy of image is focused on the lower frequency sub-band LL, and the highfrequency sub-band $\mathrm{HH}$ incorporates the edges and textures of the image where easily can be removed by low pass filter. However, the middle-frequency sub-band LH and HL are most suitable for embedding 
watermark into. So after decomposing the cover image (I) into 3-level LWT, one of the middle frequency sub-bands ( $\mathrm{LH})$ is selected to embed the watermark into it by adding its singular value with singular value of the watermark multiplied by MSFs, as shown in flowchart 1 . Furthermore, determining the MSFs values is complex problem which can be shown as optimization problem which is solved using MOABCO. To increase the security of the proposed system, before the hiding process, the original watermark is encrypted by using the chaotic map, as shown in Figure 6.

The watermark extraction procedure is an inverse of the embedding technique. Because the proposed watermark scheme is a not-blind extraction strategy, the extraction process needs information on the original image. In addition, the extracted watermark is chaotically encrypted, so it must be decrypted chaotically to get the watermark image. as shown in Figure 7.

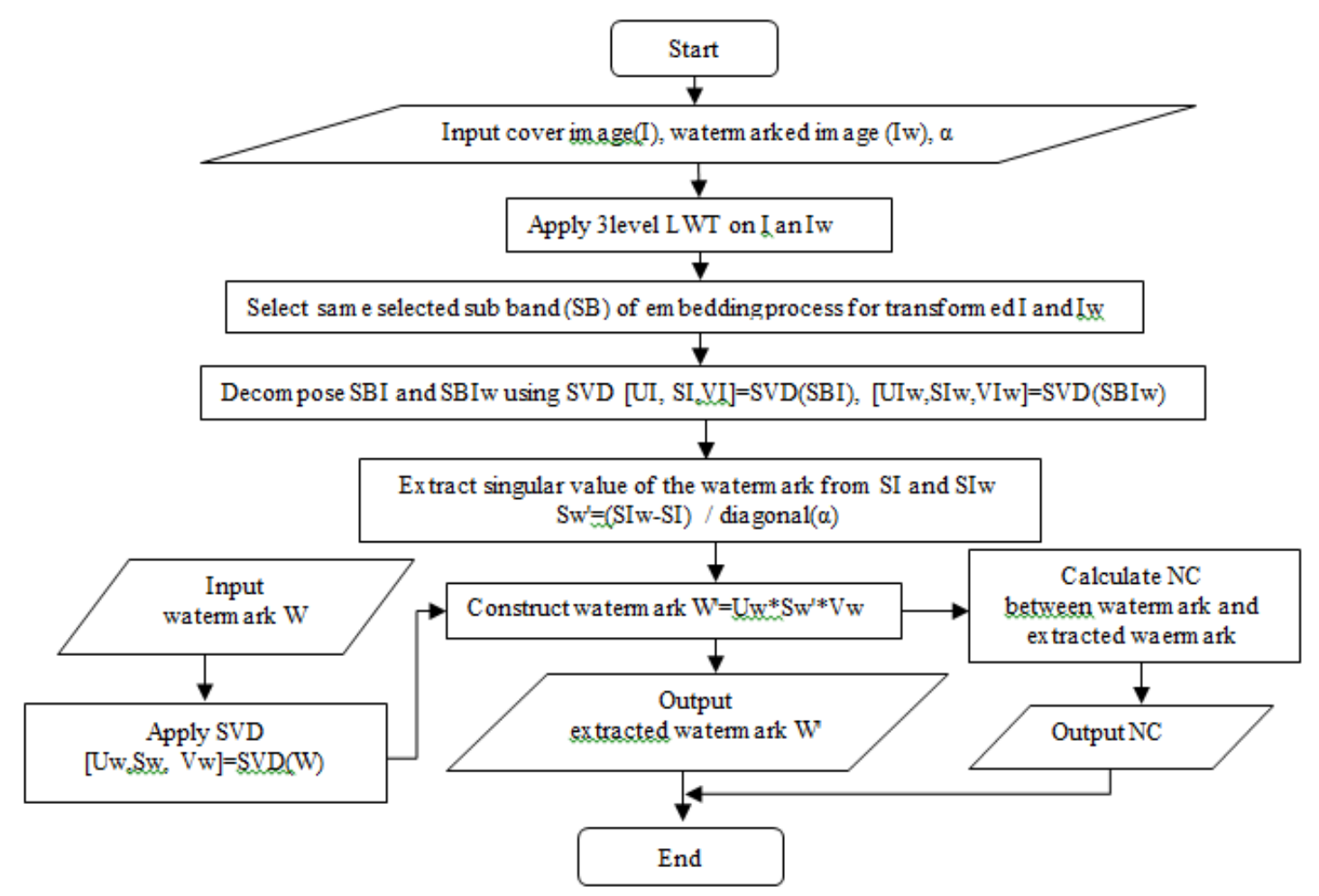

Figure 6. Flowchart of embedding process

The proposed scheme uses MSF instead of SSF to improve visual quality and to enhance the robustness of the watermarking scheme. The determination of the optimal values for MSFs of watermarking can be viewed as an optimization problem, therefore MOABC optimization algorithm is used.

In $\mathrm{MOABC}$ there are only onlooker bees, the algorithm steps are illustrated as follow.

a) Step 1: Initialize the food source positions xi randomly

where $x_{i}=\left\{x_{i 1}, x_{i 2}, \ldots, x_{i D}\right\}$ and $i=1,2, \ldots . . N S$.

Where NS is the number of solutions.

b) Step 2: Evaluate the fitness of initialized solutions.

c) Step 3: Store the infusions in the outside archive EA.

d) Step 4: For every onlooker bee $x_{i}$.

Haphazardly choose a solution $\mathrm{k}$ from EA, where $\mathrm{k} \neq \mathrm{i}$.

Create a new infusion $v_{i}$ by using expression (4)

Calculate the fitness of the new solution.

Employ a greedy selection

End for

e) Step 5: Evaluate the fitness of EA

f) Step 6: If the sum of infusions in EA surpass NS, employ crowding distance to dismiss crowded populace depending on fitness.

g) Step 7: Find the best bee in EA, the one having smallest fitness. 


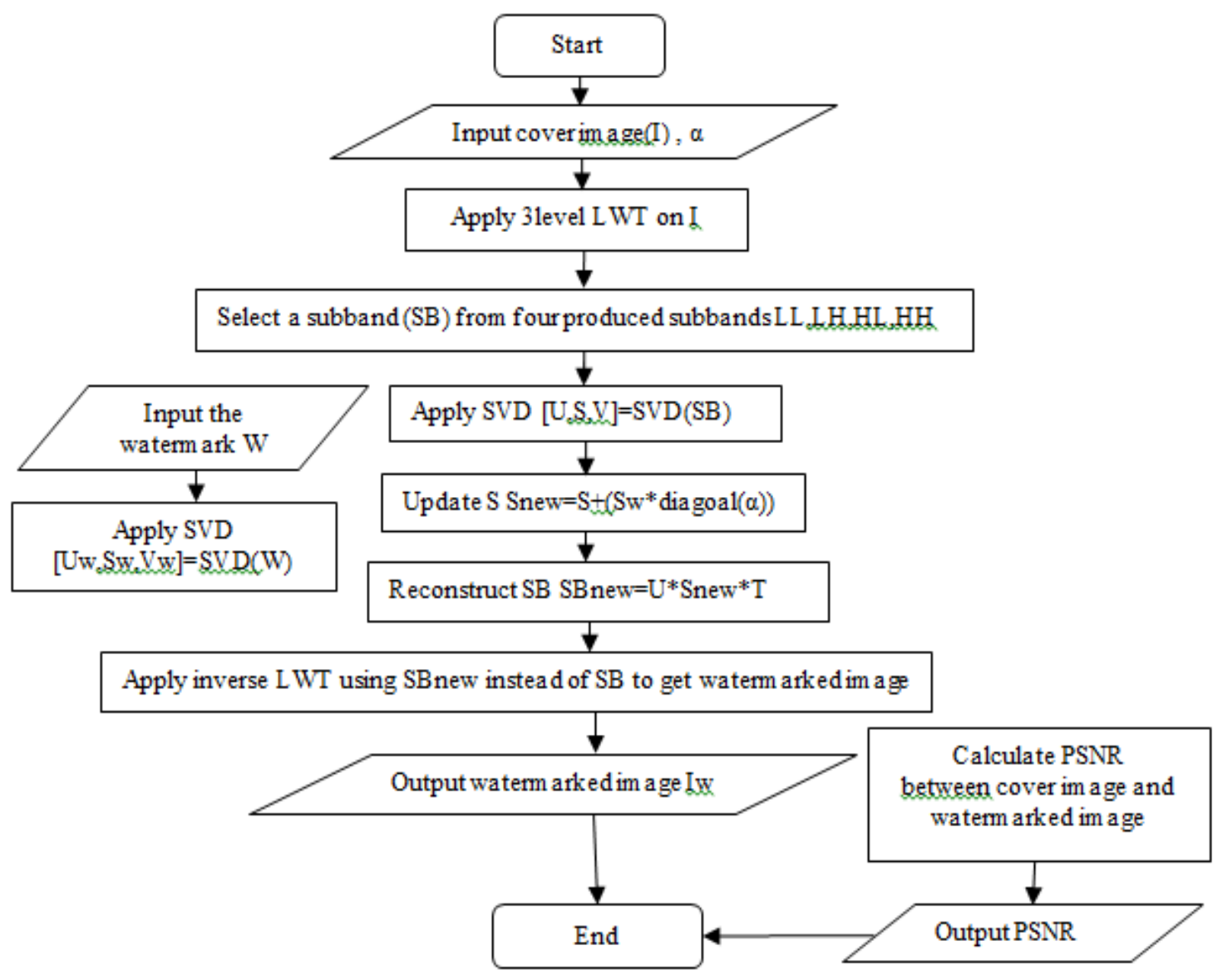

Figure 7. Flowchart of extracting process

To make the proposed scheme achieve the highest transparency and robustness under various types of attacks, the evaluation of fitness function should be progressed in such a way to present MSFs which have more resistance against attacks. If the original cover image has been introduced as I, the watermarked image as Iw and the watermark as W, then the evaluation of the fitness function of MSFs $(\alpha)$ can be illustrated in the following instruction:

1) Produce the watermarked image Iw by embedding the watermark W to the cover image I, using the embedding process.

2) Calculate the normalized correlation between I and Iw, i.e. NC (I, Iw)

3) Extract watermark $W^{\prime}$ from the watermarked image using the extraction process.

4) Calculate the normalized correlation between $\mathrm{W}$ and $\mathrm{W}^{\prime}$, i.e. $\mathrm{NC}\left(\mathrm{W}, \mathrm{W}^{\prime}\right)$

5) Apply $\mathrm{T}$ watermark attacks upon the watermarked image Iw to create $\mathrm{T}$ attacked watermarked image Îw.

6) By using the extraction procedure, separate the watermarks $\hat{W} i$ from the attacked watermarked images Îw. Where $\mathrm{i}=\{1,2, \ldots, \mathrm{T}\}$.

7) Calculate the normalized correlation between original watermark $\mathrm{W}$ and the set of extracted watermarks from attacked watermarked images.

8) Construct a vector of fitness values, defined as:

$$
\mathbf{F}(\mathrm{i})=\left(\begin{array}{c}
\frac{1}{\mathrm{NC(L,IW)}} \\
\frac{1}{N C\left(W, W^{\prime \prime}\right)} \\
\frac{1}{N C\left(W, \hat{W}_{2}\right)} \\
\vdots \\
\frac{1}{\mathrm{NC}\left(\mathrm{W}, \hat{W}_{\tau}\right)}
\end{array}\right)
$$

9) Evaluate the vector of fitness values according to the exponentially weighted method for multi-objective optimization: 


$$
F_{f i t}=\sum_{i=1}^{T+2}\left(e^{p \cdot w}-1\right) \cdot e^{p \cdot(F(i)-F o)}
$$

Where $w, p, F_{0}$ are positive constants. In experiments, the best results have been found when $w=2, p=3, F_{0}=5$.

\section{EXPERIMENTAL RESULTS}

This section presents and explores the outcomes of the proposed scheme, which are a succession of experiments that were applied to evaluate the operation of MOABC for finding the best multiple scaling factors. In the experimental results six grayscale images with size 512x512 with three formats (JPG, BMP, TIFF) are utilized as original (host) images and the watermark image is a 64x64 binary (black and white) image as shown in Figure 8 and Figure 9, respectively. To illustrate the effectiveness of the proposed scheme, the PSNR is used to estimate the imperceptibility between the watermarked image and original image, while the NC is used to find out the similarity of the original watermark and extracted watermark. The robustness of the proposed scheme is proved by applying varied types of image processing attacks on watermarked images then extracting the watermark from them.
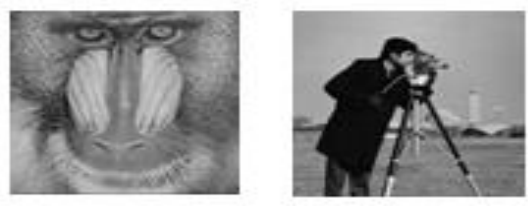

Baboon.bmp

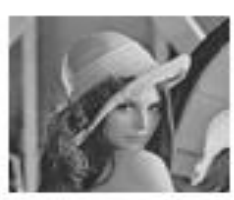

Lena.jpeg
Cameraman.jpeg

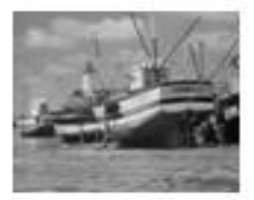

Boat.tiff

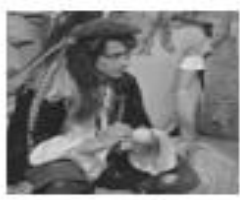

Man.bmp

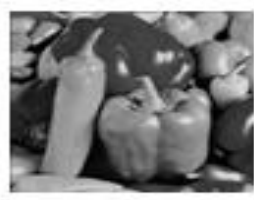

Peppers.tiff

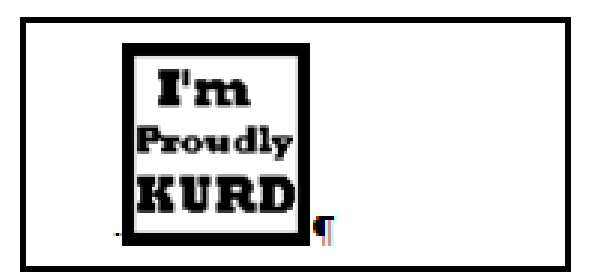

Figure 9. Binary image

Figure 8. Samples of utilized original images

In Table 1, the peak signal to noise ratios between the actual and the watermarked images, PSNR (I, IW), and the normalized correlation between the watermark and extracted watermark, $\mathrm{NC}(\mathrm{W}, \hat{\mathrm{W}})$ are shown. For examination of the robustness of the suggested scheme, the watermarked image was attacked by 12 different types of attacks incorporation into MOABC based watermarking (i.e. $\mathrm{T}=10$ ). The applied attacks are adding noise (salt \& pepper, speckle, Poisson and gaussian), cropping, sharpening, scaling, histogram equalization, quantization, and Gaussian filtering. In Table 2 the results of the testes for all six images are shown in detail after applying attacks. For the comparison reason, results of the algorithm in [15] are used, which is also a multi-objective based watermarking algorithm that uses multi-objective ant colony optimization to find the best values for MSFs. The same original images and same watermarks are used with the same size. Table 3 shows the comparison outcomes connecting them.

Table 1. Results of watermarking images

\begin{tabular}{ccc}
\hline Images & PSNR & NC \\
\hline Lena & 53.5755 & 1.0000 \\
Baboon & 52.7260 & 1.0000 \\
Cameraman & 53.0909 & 1.0000 \\
Boat & 53.2531 & 1.0000 \\
Peppers & 51.3444 & 1.0000 \\
Man & 48.4382 & 1.0000 \\
\hline
\end{tabular}


Table 2. Results of attacked watermarked images and extructed watermark

\begin{tabular}{|c|c|c|c|c|c|c|c|c|c|c|c|c|}
\hline \multirow[b]{2}{*}{ Attacks } & \multicolumn{2}{|c|}{ Lena } & \multicolumn{2}{|c|}{ Baboon } & \multicolumn{2}{|c|}{ Cameraman } & \multicolumn{2}{|c|}{ Boat } & \multicolumn{2}{|c|}{ Peppers } & \multicolumn{2}{|c|}{ Man } \\
\hline & $\begin{array}{l}\text { PSNR } \\
\text { (I,IW) }\end{array}$ & $\begin{array}{c}\mathrm{NC} \\
(\mathrm{W}, \mathrm{W})\end{array}$ & $\begin{array}{l}\text { PSNR } \\
\text { (I,IW) }\end{array}$ & $\begin{array}{c}\mathrm{NC} \\
(\mathrm{W}, \mathrm{W})\end{array}$ & $\begin{array}{l}\text { PSNR } \\
(\mathrm{I}, \mathrm{IW})\end{array}$ & $\begin{array}{c}\mathrm{NC} \\
(\mathrm{W}, \mathrm{W})\end{array}$ & $\begin{array}{l}\text { PSNR } \\
(\mathrm{I}, \mathrm{IW})\end{array}$ & $\begin{array}{c}\mathrm{NC} \\
(\mathrm{W}, \mathrm{W})\end{array}$ & $\begin{array}{l}\text { PSNR } \\
(\mathrm{I}, \mathrm{IW})\end{array}$ & $\begin{array}{c}\mathrm{NC} \\
(\mathrm{W}, \mathrm{W})\end{array}$ & $\begin{array}{l}\text { PSNR } \\
(\mathrm{I}, \mathrm{IW})\end{array}$ & $\begin{array}{c}\mathrm{NC} \\
(\mathrm{W}, \mathrm{W})\end{array}$ \\
\hline Salt \&pepper noise & 31.78 & 0.9832 & 25.02 & 0.9501 & 25.05 & 0.7831 & 25.41 & 0.9188 & 24.04 & 0.7936 & 24.95 & 0.9202 \\
\hline Speckle noise & 35.25 & 0.9346 & 34.76 & 0.9182 & 35.74 & 0.9994 & 35.25 & 0.9898 & 35.57 & 0.9834 & 35.70 & 0.9976 \\
\hline Poisson noise & 26.83 & 0.8757 & 26.43 & 0.9329 & 27.37 & 0.8152 & 26.97 & 0.8711 & 27.01 & 0.9481 & 27.11 & 0.9589 \\
\hline Gaussian noise & 16.87 & 0.9119 & 16.53 & 0.9089 & 17.51 & 0.9151 & 17.19 & 0.8855 & 16.48 & 0.9288 & 16.73 & 0.9021 \\
\hline Cropping & 29.47 & 0.9804 & 31.02 & 0.9938 & 27.20 & 0.9889 & 28.12 & 0.9802 & 30.77 & 0.9884 & 32.87 & 0.9992 \\
\hline Sharpening & 27.22 & 0.9194 & 17.40 & 0.3417 & 25.37 & 0.9745 & 22.78 & 0.5399 & 24.33 & 0.9512 & 23.14 & 0.7384 \\
\hline Scaling & 53.57 & 1.0000 & 52.72 & 1.0000 & 35.09 & 1.0000 & 53.25 & 1.0000 & 51.34 & 1.0000 & 48.43 & 1.0000 \\
\hline Histogram equalization & 16.24 & 0.9314 & 14.81 & 0.9539 & 17.41 & 0.8682 & 15.03 & 0.8986 & 15.80 & 0.9027 & 15.50 & 0.9543 \\
\hline Quantization & 45.26 & 0.9748 & 44.88 & 0.9994 & 45.36 & 0.9491 & 45.71 & 1.0000 & 44.35 & 0.9977 & 43.74 & 1.0000 \\
\hline Gaussian filtering & 33.53 & 0.0013 & 23.84 & 0.0010 & 28.99 & 0.0023 & 30.31 & 0.0571 & 30.94 & 0.0015 & 29.63 & 0.1414 \\
\hline
\end{tabular}

Table 3. Comparison to the reference [15]

\begin{tabular}{ccccc}
\hline \multirow{2}{*}{ Images } & \multicolumn{3}{c}{ PSNR } & \multicolumn{2}{c}{ NC } \\
& Proposed Scheme & Algorithm From [15] & Proposed Scheme & Algorithm From [15] \\
\hline Lena & 50.3469 & 47.718 & 1.0000 & 1.0000 \\
Baboon & 52.7575 & 52.379 & 1.0000 & 1.0000 \\
Cameraman & 51.1876 & 48.902 & 1.0000 & 1.0000 \\
Boat & 55.7951 & 54.810 & 1.0000 & 1.0000 \\
Peppers & 46.1877 & 48.097 & 1.0000 & 1.0000 \\
Man & 50.6255 & 50.181 & 1.0000 & 1.0000 \\
\hline
\end{tabular}

\section{CONCLUSION}

In this paper, we proposed an algorithm for watermark embedding and watermark extraction Embedding encrypted watermark to high frequency subbands allows high performance watermark extraction. In this paper a semi-blind watermarking algorithm is presented which is established on lifting wavelet transform and singular value decomposition. Multiple scaling factors are used instead of single scaling factor to get the highest probable transparency and robustness together. The MOABC algorithm is used to determine potential MSFs. High transparency is obtained since no difference can be noticed after embedding watermark to the original image. Good robustness against various types of attacks is noticed. In terms of performance and PSNR. By increasing the levels of decomposition for the watermarked image, the resistance against the attacks and the quality of extracted watermark can be improved.

\section{REFRENCE}

[1] S. Priya, B. Santhi, and P. Swaminathan, "Image watermarking techniques-a review," Research Journal of Applied Sciences, Engineering and Technology, vol. 4, no. 14, pp. 2251-2254, 2012.

[2] Brifcani, A. M. A., \& Al-Bamerny, J. N. "Image compression analysis using multistage vector quantization based on discrete wavelet transform". In 2010 International Conference on Methods and Models in Computer Science (ICM2CS-2010), pp. 46-53, IEEE, 2010.

[3] Prajwalasimha, S. N., Chethan Suputhra, S., \& Mohan, C. S. "Performance analysis of DCT and successive division based digital image watermarking scheme", Indonesian Journal of Electrical Engineering and Computer Science (IJEECS), vol. 15, no. 2, pp. 750-757, 2019.

[4] Abdulqader, D. M., Abdulazeez, A. M., \& Zeebaree, D. Q. "Machine Learning Supervised Algorithms of Gene Selection: A Review", Machine Learning, vol. 62, no. 3, 2020.

[5] F. Hartung and M. Kutter, "Multimedia watermarking techniques," Proceedings of the IEEE, vol. 87, no. 7, pp. 1079-1107, 1999.

[6] I. M. N. Adeen, A. M. Abdulazeez, and D. Q. Zeebaree, "Systematic Review of Unsupervised Genomic Clustering Algorithms Techniques for High Dimensional Datasets: A Review", Machine Learning, vol. 62, no. 3, 2020.

[7] Sulaiman, D. M., Abdulazeez, A. M., Haron, H., \& Sadiq, S. S. "Unsupervised Learning Approach-Based New Optimization K-Means Clustering for Finger Vein Image Localization". In 2019 International Conference on Advanced Science and Engineering (ICOASE), pp. 82-87. IEEE, 2019.

[8] Zeebaree, D. Q., Haron, H., Abdulazeez, A. M., \& Zebari, D. A. "Trainable Model Based on New Uniform LBP Feature to Identify the Risk of the Breast Cancer". In 2019 International Conference on Advanced Science and Engineering (ICOASE), pp. 106-111. IEEE, 2019.

[9] P. Singh and R. Chadha, "A survey of digital watermarking techniques, applications and attacks," International Journal of Engineering and Innovative Technology (IJEIT), vol. 2, no. 9, pp. 165-175, 2013.

[10] Al-Yousuf, F. Q. A., \& Din, R. "Review on secured data capabilities of cryptography, steganography, and watermarking domain", Indonesian Journal of Electrical Engineering and Computer Science (IJEECS), vol. 17, no. 2, pp. 1053-1059, 2020. 
[11] Zebari, D. A., Haron, H., Zeebaree, D. Q., \& Zain, A. M. "A Simultaneous Approach for Compression and Encryption Techniques Using Deoxyribonucleic Acid". In 2019 13th International Conference on Software, Knowledge, Information Management and Applications (SKIMA), pp. 1-6. IEEE, 2019.

[12] Alias, N., \& Ernawan, F. "Multiple watermarking technique using optimal threshold". Indonesian Journal of Electrical Engineering and Computer Science (IJEECS), vol. 18, no. 1, pp. 368-376, 2020.

[13] Zeebaree, D. Q., Abdulazeez, A. M., Hassan, O. M. S., Zebari, D. A., \& Saeed, J. N. "Hiding Image by Using Contourlet Transform", vol. 83, pp. 16979-16990, 2020.

[14] L. K. Saini and V. Shrivastava, "A survey of digital watermarking techniques and its applications," arXiv preprint arXiv:1407.4735, 2014.

[15] N. Harish, B. Kumar, and A. Kusagur, "Hybrid robust watermarking techniques based on DWT, DCT, and SVD," International Journal of Advanced Electrical and Electronics Engineering, vol. 2, no. 5, pp. 137-143, 2013.

[16] M. I. Khan, M. Rahman, M. Sarker, and I. Hasan, "Digital Watermarking for Image AuthenticationBased on Combined DCT, DWT and SVD Transformation," arXiv preprint arXiv:1307.6328, 2013.

[17] M. S. Islam and U. P. Chong, "A digital image watermarking algorithm based on DWT DCT and SVD," International Journal of Computer and Communication Engineering, vol. 3, no. 5, p. 356, 2014.

[18] H. Saxena, P. Saxena, and S. Rastogi, "DWT-DCT-SVD based semi-blind reference image watermarking scheme using trigonometric function," International Journal of Conceptions on Computing and Information Technology, vol. 2, no. 2, pp. 14-18, 2014.

[19] S. G. Kejgir and M. Kokare, "Lifting wavelet transform with singular value decomposition for robust digital image watermarking," International Journal of Computer Applications, vol. 39, no. 18, pp. 10-18, 2012.

[20] A. Tun and Y. Thein, "Digital image watermarking scheme based on LWT and DCT," International Journal of Engineering and Technology, vol. 5, no. 2, p. 272, 2013.

[21] Y. Chen, W. Yu, and J. Feng, "A reliable svd-dwt based watermarking scheme with artificial bee colony algorithm," International Journal of Digital Content Technology and its Applications, vol. 6, no. 22, p. 430, 2012.

[22] W. Sweldens, "The lifting scheme: A construction of second-generation wavelets," SIAM journal on mathematical analysis, vol. 29, no. 2, pp. 511-546, 1998.

[23] C.-C. Lai, "A digital watermarking scheme based on singular value decomposition and tiny genetic algorithm," Digital Signal Processing, vol. 21, no. 4, pp. 522-527, 2011.

[24] V. Aslantas, "Optimal SVD based robust watermarking using differential evolution algorithm," in Proceedings of the world Congress on Engineering, vol. 1, pp. 2-4, 2008.

[25] K. Loukhaoukha, J.-Y. Chouinard, and M. H. Taieb, "Optimal image watermarking algorithm based on LWT-SVD via multi-objective ant colony optimization," Journal of Information Hiding and Multimedia Signal Processing, vol. 2, no. 4, pp. 303-319, 2011.

[26] W. Zou, Y. Zhu, H. Chen, and B. Zhang, "Solving multiobjective optimization problems using artificial bee colony algorithm," Discrete dynamics in nature and society, vol. 2011, 2011.

[27] K. Loukhaoukha and J.-Y. Chouinard, "Hybrid watermarking algorithm based on SVD and lifting wavelet transform for ownership verification," in 2009 11th Canadian Workshop on Information Theory, pp. 177-182: IEEE, 2009.

[28] G. Uytterhoeven, D. Roose, and A. Bultheel, "Integer wavelet transforms using the lifting scheme," in 3rd World Multiconference on Circuits, Systems, Communications and Computers, Date: 1999/07/04-1999/07/08, Location: Athens, Greece, 1999, pp. 198-200: IEEE/IMACS/OTE.

[29] M. Janardan and K. Babu, "An efficient architecture for 3-D lifting-based discrete wavelet transform," Int. J. Comp. Tech. Appl, vol. 2, no. 5, pp. 1439-1458, 2011.

[30] Z. Prusa and P. Rajmic, "Real-time lifting wavelet transform algorithm," J. Signal Process, vol. 2, no. 3, pp. 53-59, 2011.

[31] K. Deb, A. Pratap, S. Agarwal, and T. Meyarivan, "A fast and elitist multiobjective genetic algorithm: NSGA-II," IEEE Transactions on Evolutionary Computation, vol. 6, no. 2, pp. 182-197, 2002.

[32] R. A. Sadek, "SVD based image processing applications: state of the art, contributions and research challenges," arXiv preprint arXiv:1211.7102, 2012.

[33] R. Liu and T. Tan, "An SVD-based watermarking scheme for protecting rightful ownership," IEEE Transactions on Multimedia, vol. 4, no. 1, pp. 121-128, 2002.

[34] N. Venkatram, L. Reddy, P. Kishore, G. Fields, G. D. Vaddeswaram, and A. Pradesh, "Blind medical image watermarking with LWT-SVD for telemedicine applications," Image, vol. 20, p. 23, 2014.

[35] I. J. Cox, J. Kilian, F. T. Leighton, and T. Shamoon, "Secure spread spectrum watermarking for multimedia," IEEE Transactions on Image Processing, vol. 6, no. 12, pp. 1673-1687, 1997.

[36] D. Karaboga, "An idea based on honey bee swarm for numerical optimization," Technical report-tr06, Erciyes university, engineering faculty, computer, 2005.

[37] D. T. Pham, A. Ghanbarzadeh, E. Koç, S. Otri, S. Rahim, and M. Zaidi, "The bees algorithm-a novel tool for complex optimisation problems," in Intelligent production machines and systems: Elsevier, pp. 454-459, 2006.

[38] Ahmed, J. A., \& Brifcani, A. M. A. "A new internal architecture based on feature selection for holonic manufacturing system". International Journal of Mechanical, Aerospace, Industrial, Mechatronic and Manufacturing Engineering, vol. 2, no. 8, p. 1431, 2015.

[39] Zeebaree, D. Q., Haron, H., Abdulazeez, A. M., \& Zebari, D. A. "Machine learning and Region Growing for Breast Cancer Segmentation". In 2019 International Conference on Advanced Science and Engineering (ICOASE), pp. 8893. IEEE, 2019. 
[40] Hassan, O. M. S., Abdulazeez, A. M., \& TIRYAKİ, V. M. "Gait-based human gender classification using lifting 5/3 wavelet and principal component analysis". In 2018 International Conference on Advanced Science and Engineering (ICOASE), pp. 173-178. IEEE, 2018.

[41] Sengupta, S., Basak, S., \& Peters, R. A. "Particle Swarm Optimization: A survey of historical and recent developments with hybridization perspectives". Machine Learning and Knowledge Extraction, vol. 1, no. 1, pp. 157-191, 2019.

[42] S.-J. Xue and W. Wu, "Scheduling workflow in cloud computing based on hybrid particle swarm algorithm," TELKOMNIKA Indonesian Journal of Electrical Engineering, vol. 10, no. 7, pp. 1560-1566, 2012.

[43] P. Melin, V. Herrera, D. Romero, F. Valdez, and O. Castillo, "Genetic optimization of neural networks for person recognition based on the Iris," TELKOMNIKA Indonesian Journal of Electrical Engineering, vol. 10, no. 2, p. 309, 2012.

[44] A. M. Ali, M. Ebrahim, and M. M. Hassan, "Automatic voltage generation control for two area power system based on particle swarm optimization," Indonesian Journal of Electrical Engineering and Computer Science (IJEECS), vol. 2, no. 1, p. 132, 2016.

[45] Ahmed, F. Y., Shamsuddin, S. M., \& Hashim, S. Z. M. "Improved SpikeProp for using particle swarm optimization". Mathematical Problems in Engineering, 2013.

[46] Khudher, I. M., \& Ibrahim, Y. I. "Swarm intelligent hyperdization biometric". Indonesian Journal of Electrical Engineering and Computer Science (IJEECS), vol. 18, no. 1, pp. 385-395, 2020. 